\title{
RoutePlanner Model - Support for Financial Brokers Using Visualization to Enhance Insight into Information
}

\author{
Patricia M. Gouws and M. R. (Ruth) de Villiers \\ School of Computing, University of South Africa, South Africa,
}

\author{
gouwspm@unisa.ac.za; dvillmr@unisa.ac.za
}

\begin{abstract}
This paper considers the needs of financial brokers seeking to use information visualization to transform raw data in a data warehouse into enhanced insight, so as to identify marketing opportunities and better inform their clients. We introduce a model of the visualization process, called RoutePlanner, which was developed from a theoretical perspective. RoutePlanner comprises guidelines and stages for the identification of the data to be visualized, the selection of appropriate visualization methods, and the evaluation of the visualizations. In a collaborative process with a data expert and a visualization expert, the domain experts (brokers) were oriented in the use of RoutePlanner. They evaluated each stage of RoutePlanner positively, as well as the visualization process as a whole. The selected visualizations were successful in terms of the enhanced insight they provided, and the brokers who participated in the study were able to better inform and advise their clients in financial planning sessions.
\end{abstract}

Key terms: Domain analysis, evaluation, guidelines, identification, selection, visualization methods, information visualization.

\section{Introduction}

This paper describes a study conducted in the application area of financial brokerage, where the focus area is the process of information visualization. The research, which originates from a masters degree study by the primary author, relates to ways of supporting financial brokers in using data warehousing and information visualization so that the brokers can be better informed and, in turn, also inform their clients.

We present a model of the visualization process that identifies relevant data, selects and uses visualization methods to transform raw data in a warehouse to representations, and then evaluates the visualizations that provide enhanced insight, using guidelines for each stage of the process. The stages of the visualization process are described and the findings are given of surveys among the financial brokers both before and

Material published as part of this publication, either on-line or in print, is copyrighted by the Informing Science Institute. Permission to make digital or paper copy of part or all of these works for personal or classroom use is granted without fee provided that the copies are not made or distributed for profit or commercial advantage AND that copies 1) bear this notice in full and 2) give the full citation on the first page. It is permissible to abstract these works so long as credit is given. To copy in all other cases or to republish or to post on a server or to redistribute to lists requires specific permission and payment of a fee. Contact 0HPublisher@InformingScience.org to request redistribution permission. after exposure to the visualization process.

\section{Background}

Some key concepts of the study are described, after which we explain the context of the study in terms of the realworld problem and the target group. 


\section{Data Warehousing}

According to a formal definition by Kimball (1996), a data warehouse is a repository of temporal, multi-dimensional, multi-variant, structured and abstract data, ordered in terms of facts and dimensions. He advocates the use of warehouses to represent organizational data. The data must be meaningful and interpretable and should provide the user with enhanced insight. Data warehouses store large volumes of data, with further data being added at regular intervals. Whereas a database contains only the most recent values, a data warehouse contains multiple data snapshots, thus allowing for the analysis of data trends and historical data.

There are two approaches to the analysis of warehouse data, namely the automated and the manual approach. The automated option uses data mining algorithms to extract information. The manual option, which is the approach adopted in the present study, requires holistic userexploration and interpretation of the data. This exploration process can be guided and enhanced by visual presentation of the data, so as to discover data patterns, outlier values and data clusters. These findings, in turn, must be used to generate meaningful information that can be applied in the real-world context.

\section{Visualization}

Information visualization (IV) is the use of computer-supported interactive visual representations of abstract data to amplify cognition (Card, Shneiderman \& MacKinlay, 1999). The purpose of visualization is the exploration of data and the presentation of information so that data owners can better understand their data and use any volume of raw data, including large volumes (Ward \& Theroux, 1997; Wiss, Carr \& Jonsson, 1998). In short, according to Saraiya, North and Duca (2005), the main purpose of visualization is the generation of insight.

\section{Relationship Between Data Warehousing and Visualization}

Information visualization, as opposed to scientific visualization, aims to visualize abstract data with no inherent structure. Such data may have no intuitive or formal visual representation, and IV is therefore an appropriate strategy for its exploration. A large collection of visualization methods (VMs) is available, with different methods serving varying functions. Shneiderman (1996) refers to IV in terms of seven abstract tasks that are included in his visual 'information-seeking mantra', namely: overview, zoom, filter, details-on-demand, relate, history and extract.

\section{Real-World Problem}

Under the present system, financial brokers in the target group use a financial needs analysis (FNA) program to access and to manipulate a database containing the most recent financial data for each of the clients in his/her client portfolio. The overview and analysis process may be conducted by considering a client's resources and requirements, usually during a client-broker consultation, where the broker selects that specific client's information from the portfolio, extracts a particular unit of data for analysis (e.g. pension, estate management or financial planning) and then views the client's details for that unit. For the broker to view the data of all clients in a portfolio, a limited number of fixed queries are included in the FNA program, the results of which are presented as lists. The database does not store historical data, thus the broker sees only the data relating to the current status of each client. Thus, there are major limitations on the insights gained from the data in its present format and structure, using the current FNA program for representation of results. 


\section{Envisaged New Approach}

Using the present FNA program, a broker, is unable to gain a holistic or historical overview of the data within the client portfolio, and is neither able to inform his/her clients optimally, nor to identify client needs or marketing opportunities easily. To address this problem, a new approach was designed to support brokers in using IV to inform themselves and their clients. By generating snapshots of the data in a stereotypical broker's database at regular intervals and loading these snapshots into a data warehouse, a prototype warehouse was developed. The visual presentation and exploration of this data can facilitate the generation of insights and provide information for both the broker and the broker's clients. The brokers, who are considered domain experts in their profession, may not be experts in visualization nor in data or information technology. They therefore required orientation and training in the new approach. Small-group orientation sessions were conducted, encouraging collaboration between the domain experts, a data expert and an information-visualization expert.

\section{Research Design and Methodology}

\section{Research Questions}

The study sets out to answer the following questions:

- Does the system currently used by the target group of brokers support users in translating raw data into insight?

- What are appropriate stages in the visualization process of translating data into insight and what should each stage entail?

- What guidelines can help users apply this visualization process?

- Can this visualization process be used by brokers to gain adequate insight?

The first and last questions are answered by the findings of an empirical study, and answers to the second and third were extracted from the literature. The work reported in this paper is therefore a combination of empirical research and non-empirical research, as described in Mouton's (2003) research map. This section briefly outlines the research approaches and methods, prior to elaborating each in the next section.

\section{Initial Empirical Research - Identification of Need}

Initial empirical research, termed the 'identification-of-need' study, used a questionnaire to obtain qualitative and quantitative data to establish a profile of typical brokers and their client portfolios. This determined the level of awareness of visualization and its benefits, and determined whether the brokers could clearly define the data required, so as to best identify marketing opportunities and inform clients. A further purpose was to ascertain their attitudes to the introduction and use of visualizations with an associated learning curve. This study indicated a clear need for change.

\section{Non-Empirical Research - Literature-Based Model Building}

This major area of the work combined two of Mouton's (2003) non-empirical forms of research, namely: model building based on a literature study. This involved the development of a model of the visualization process, called RoutePlanner. This model is a stage-by-stage process to support brokers in using existing readily-available visualization methods to generate insight into the identification of new marketing opportunities, and to better inform and advise their clients. Following a major literature study, RoutePlanner was developed using principles and methods based on theories and precedents from the literature. 


\section{Subsequent Empirical Research - Evaluation of Model}

To validate this need and implement the proposed model, a group of brokers was identified to participate in a study. A questionnaire, involving mainly Likert-scale data, was used to establish the awareness of visualization and the visualization process of the broker, both before and after the use of the model of the visualization process during several small-group sessions. A data warehouse was designed, and populated with a snapshot of stereo-typical broker data. During the small-group sessions, the process embodied in the RoutePlanner Model was introduced, demonstrated and used.

These collaborative sessions between the domain expert (the broker), a data expert and a visualization expert (in some of the groups, the data expert and visualization expert were one-and-thesame person) included evaluations in the form of observations by the researcher and questionnaires completed by the brokers. These evaluations ascertained the value of each stage of the visualization process, as well as the application of RoutePlanner as a whole. The ability of a domain expert to facilitate the translation of data into insight using the visualization process depends on communication. Van Wijk (2006), Kao and Ma (2000) and Duke, Brodlie, Duce and Herman (2005) advocate considerable collaboration between the domain expert and the visualization expert. Although in exceptional cases, the domain expert may have the required data knowledge, and thus also be the data expert, optimally there should be also be a separate data expert as a third party in the collaboration.

\section{Identification of the Need for Visualization}

The initial empirical research, an identification-of-need study, was done at a conference of financial brokers to establish their profile, to investigate their awareness of IV and VMs, to query how they identified marketing opportunities, and to ascertain attitudes to visualization. The primary researcher served as facilitator and presented a range of VMs to the 31 participants. Apart from pie-charts and bar charts, with which $84 \%$ and $81 \%$ of participants respectively were very familiar, bubble-plots and scatter-plots were less known, with only $10 \%$ describing themselves as very familiar with bubble-plots and $23 \%$ with scatter-plots. Participants gave rated responses to four statements relating to the visualization of marketing opportunities, as shown in Figure 1.

\section{Visualization of marketing opportunities $(n=31)$}

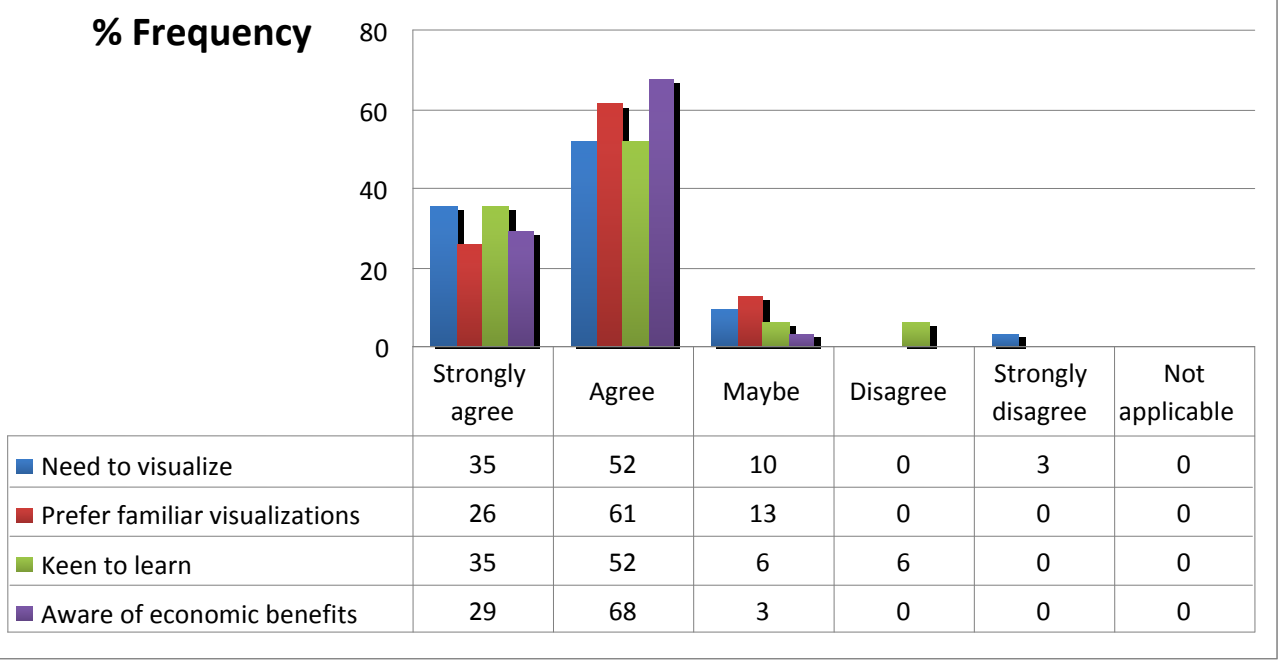

Figure 1 Visualization of marketing opportunities 
More than $80 \%$ of the participants either agreed or strongly agreed with the statements relating to a need for visualization, a preference for familiar visualizations, a keenness to learn about visualization, as well as an awareness of the economic benefits of using visualization.

To determine the participants' awareness of the process of visualization, the participants rated two further statements. These responses are shown in Figure 2.

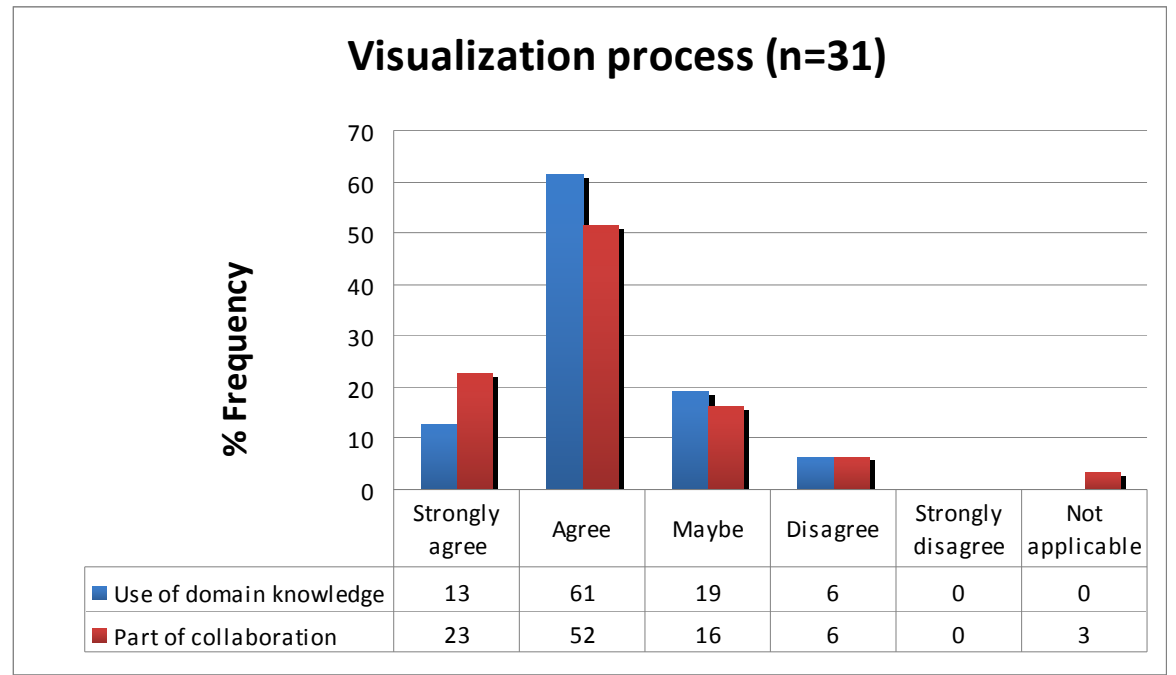

Figure 2 Visualization process

More than $70 \%$ of the participants either agreed or strongly agreed that domain knowledge was required and that the orientation into using the process of visualization should be done collaboratively with visualization experts.

\section{Literature-Based Model Building - Development of Routeplanner: A Model of the Visualization Process}

The development of a proposed model of the visualization process, named RoutePlanner, was based on a study that surveyed the literature for a theoretical assessment of the following issues:

Stages in the visualization process used for the translation of data into insight.

Guidelines to be used in the application of this process.

Application of this visualization process by brokers to translate their raw client data into insight, so as to perform their professional activities more effectively.

No single or fixed set of stages was identified in the literature for the visualization process as a path from raw data to required insight. However, a consolidated framework was proposed, which takes cognisance of various definitions and structures from the literature. We decomposed the IV process into three generic stages:

- Identification stage: An iterative stage that considers which aspects of the data should be visualized and what user tasks are required.

- Selection stage: Appropriate visualization methods must be selected.

- Evaluation stage: The selected visualizations are evaluated by the users to determine whether or not the required insight has been attained. 
The overall idea is that, for each of the data aspects identified in the identification stage, appropriate VMs should be selected from the collection to visualize this data. The selected VMs should then be implemented, taking the data formatting requirements for each of the methods into consideration. In an evaluation, these visualizations are scrutinized to decide whether they achieve their purposes. The initial use of RoutePlanner is simplified if a broker (the domain expert) does orientation sessions in collaboration with a visualization expert and a data expert.

Each stage of RoutePlanner has a set of resources in the form of templates to support the application of that stage. It is recommended that the team discuss each template, and that the details of the discussions be recorded. As brokers become familiar with the visualizations, representations and interactions, they should accumulate personal visualization literacy and expertise, which can lead, ultimately, to independent application of RoutePlanner. After sufficient collaborative orientation and training, they should be able to use the selected visualizations independently in consultation sessions with their clients.

\section{Guidelines}

To answer the third research question regarding guidelines to help the brokers in the application of the visualization process, a literature study focused, among others, on the work of Brath (1999), Mann (1999), Mann and Reiterer (2000) and Spence (2001). A set of guidelines was acquired, each of which plays a useful and relevant role in the guidance of the visualization process. For this reason, the sets of guidelines are discussed first, prior to addressing the defined stages of RoutePlanner. The guideline templates (not shown in this paper) are intended to enhance the overall focus on the problem that requires visualization. Figure 3 presents RoutePlanner's collection of guidelines.

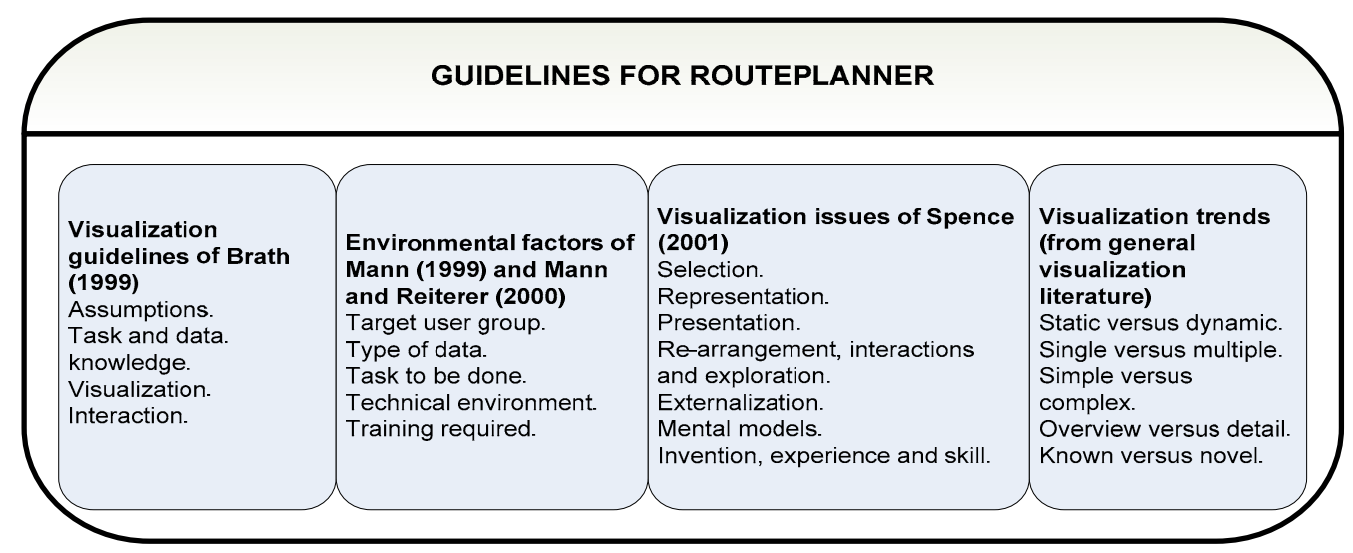

Figure 3 Guidelines in RoutePlanner

The guidelines were introduced to the brokers at the start of the small-group sessions. Guidelines are considered part of the planning prior to the identification stage, and are also referred to throughout the Route Planner process.

- Guidelines of Brath (1999): Brath's guidelines take into account prior assumptions and the definition of the visualization goal, tasks, data, visualization and interactions. His first area of guidelines requires that the users have a clear understanding of the context. Any assumptions should be explicitly noted before the visualization process and recorded in the RoutePlanner templates. Brath warns that guidelines should not be viewed as rules. There should be a goal to solve a specific problem and sufficient information should be available. Requirements must be defined in terms of the data to be visualized and the tasks to be supported. An incomplete understanding of the goals, user tasks and data can hinder the generation of an effective visualization. Brath's further guidelines identify important aspects of vis- 
ual representations and hazards to avoid. Other guidelines relate to interaction, supporting the user in manipulating visualizations and identifying patterns.

- Environmental factors of Mann (1999) and Mann and Reiterer (2000): Mann's so-called 4T environmental factors are target users, type of data, task in hand, and technical environment. Training was added by Mann and Reiterer (2000), leading to the 5T Factors. The experts should discuss each factor and record the details.

- Visualization issues of Spence (2001): To guide the actual visualization of data, Spence (2001) presents seven universal visualization issues that were identified prior to the era of computerized visualizations, but are equally relevant to electronic visualizations. These issues are the selection of data (only relevant data should be selected for visual exploration); representation (must be appropriate, involving a mental model that may differ from the user's perception); presentation; rearrangement, interactions and exploration; externalization of data; the mental models; and the invention, experience and skill of the user (all these are required to fully understand the task for which the visualization is intended). While these issues may be the basics of a successful visualization, there is no single visualization that is always the best for a certain kind of data.

- Visualization trends: From perusal of the visualization literature, the primary researcher synthesized a list of trends representing general attributes of information visualization. Each trend represents an attribute that may be implemented in more than one way and it is up to the intended users of VMs to decide which option is the more suitable for the task in hand.

As the visualization methods evolve, so too, do the associated guidelines and issues. The guidelines in Figure 3, which have been discussed above, were included in RoutePlanner to assist and direct the team of experts in their discussions prior to and during the visualization process. We support the stance of Brath (1999) that guidelines may be incomplete, evolving and universal, and that their use provides no guarantees for an effective visualization. However, guidelines comprise a body of knowledge that can be used by domain experts and data experts, who lack the specialized knowledge and skill to interpret visualizations meaningfully. Such guidelines can also be used by the visualization expert for reference purposes.

\section{Identification Stage}

Identification involves discerning study to identify which data should be visualized and to specify the goal of the visual exploration, along with the associated user tasks. The exploration starts in a data warehouse, a large volume of multi-dimensional and temporal facts and dimensions, as described in an earlier section.

RoutePlanner makes use of domain analysis (Espinosa, Hendrickson and Garret, 1999) to implement identification. Domain analysis is a systematic approach for investigating the domain where visualizations are required. It includes the domain description, analysis of the user needs, and systematic methods for gathering knowledge, such as expert interviews, literature reviews, observation and problem solving. Such analysis produces documented requirements for the visualization tasks, which may used again in the evaluation of the visualization. The major task at this stage for the participants is the completion of the identification stage template. This involves completing a questionnaire based on Espinosa et al's domain analysis. Supplementary task definitions may be guided by Shneiderman's (1996) definition of abstract tasks. OLIVE (1997) makes suggestions for visualizing multi-dimensional data (e.g. to understand the whole or part of the n-dimensional data, to find a specific item or group of items in the data) and also tasks relating to temporal data (e.g. viewing specific events, sequencing data, creating historical overviews). Kimball, Reeves, Ross and Thornthwaite (1998) offer guidance for a typical cycle of warehouse data analysis. Details of the identification stage are presented in Figure 4 below. 


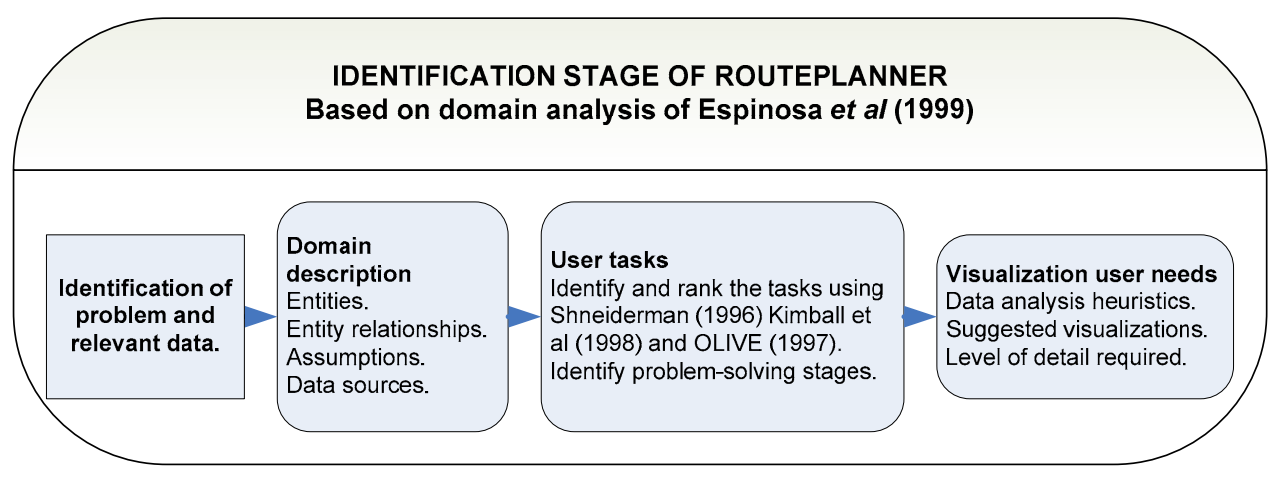

Figure 4 Identification stage of RoutePlanner

Identification of the data, goals and tasks can involve several iterations of domain analysis to ensure that the goal, data and user tasks are adequately captured. At the end of the stage, the collaborating experts should have a list of the data to be visualized. This contributes to the goal of the visualization, and the selection stage may then commence.

\section{Selection Stage}

During selection, appropriate visualization methods (VMs) are identified, either selected from a large collection of existing VMs, or created as a new, novel VM. The creation option was not included in this study. To guide the selection process, a human visualization expert may be consulted (Kao \& Ma, 2000); an automated Visualization Assistant (ViA) developed by Healey, Amant and Elhaddad (1999) may be used (currently unavailable); or the collection of visualization method taxonomies may be referred to. For this study, the latter was most suitable.

The taxonomies were presented as a comprehensive and evolving body of knowledge synthesised from standard taxonomies gleaned from the literature. The VMs were constrained by availability, specifically in the public domain or as non-commercial demonstration versions of visualization systems. This study used InfoVis Toolkit (2008), VisTree (2008) and XmdvTool (2008).

The manual approach to the selection involves consulting such taxonomies and their classification criteria, and using them for the selection of one or more appropriate VMs. The collections of VM taxonomies in RoutePlanner (see Figure 5) are:

- The task by data type taxonomy of Shneiderman (1996): An approach that classifies visualizations according to the type of data and abstract task type definitions.

- OLIVE'S data type taxonomy (1997): An extended background to Shneiderman's (1996) approach, and a useful resource for guidance.

- Taxonomy of information visualization interfaces: Linked to OLIVE. These sites provide examples of visualizations for defined data types.

- Data and visualization type, interaction and distortion taxonomy of Keim (2002): Keim classifies visual exploration methods according to data type, categorical classification of VM, and the interaction and distortion technique considered. For the visual exploration of any data type, any category of VM may be selected with any interaction and distortion technique. Techniques associated with each of the VM categories differ in the way that the data is arranged and dimensions are handled.

- Categories of visualization methods taxonomy of de Oliveira and Levkowitz (2003): In this taxonomy, VMs are classified into four categories, including the characteristics of methods within the category. 
- Complexity, content area, point of view, type of thinking and type of representation taxonomy of Lengler and Eppler (2007): This taxonomy presents VMs in a periodic table format, and is interactive, showing examples of each VM when the mouse passes over the dynamic presentation.

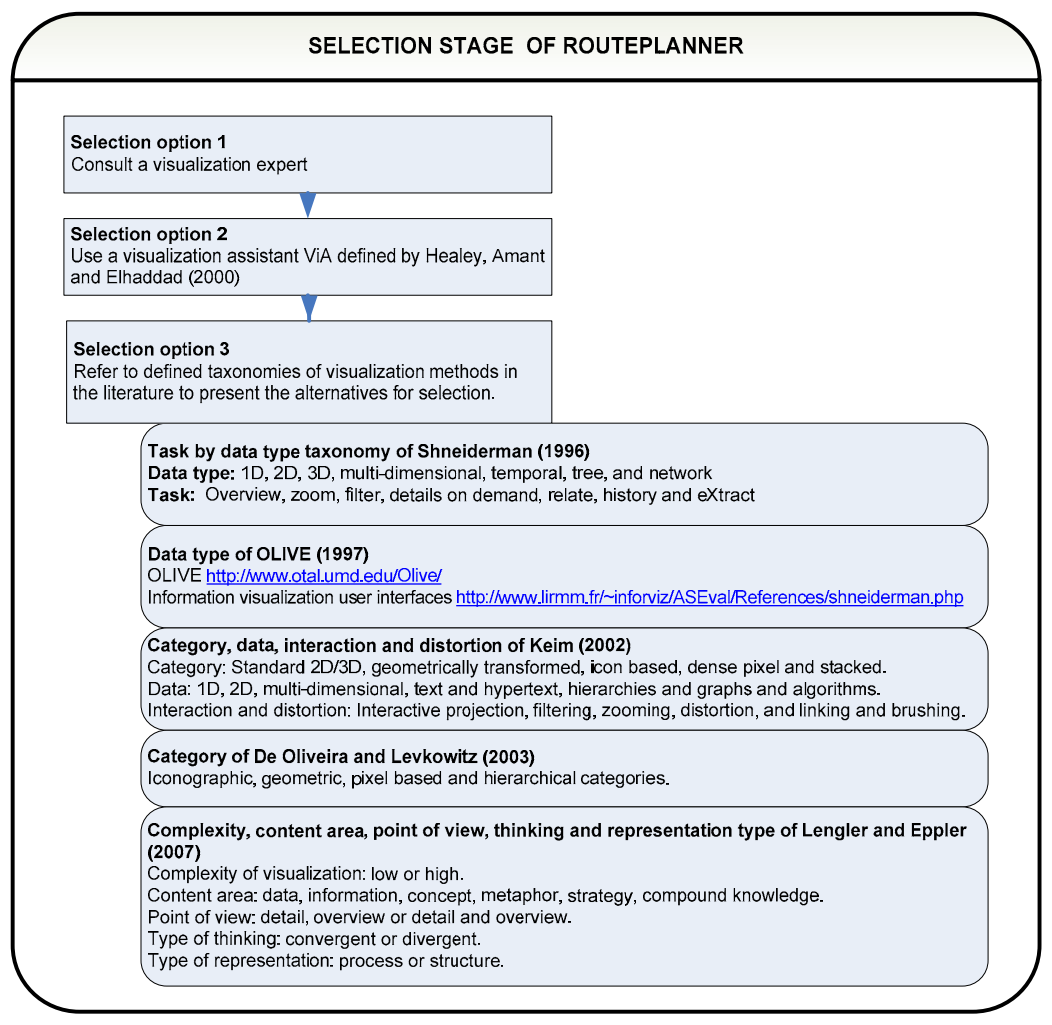

Figure 5 Selection stage of RoutePlanner

Given the warning of de Oliveira and Levkowitz (2003) that selection is a specialized, contextual process requiring experience, skill and intuition, RoutePlanner should ensure that the selection stage is supported by documentation and experience for situations where the user lacks the skill and expertise to select a specific method. For this study, the expertise is acquired in a consultative process, whereby the domain experts, in collaboration with a visualization experts and data expert, select VMs for the data in hand, bearing in mind the user tasks identified and the available VMs. Resources and references for the selection of VMs are included in RoutePlanner to guide the selection stage of the visualization process. Once the data is in the required format, the visualizations may be implemented using the selected methods.

\section{Evaluation Stage}

The visualizations of the domain data using the selected VMs need to be evaluated. The purpose of the evaluation stage is:

- Firstly, to evaluate alternative visualizations and identify the most appropriate one.

- Secondly, to identify the insight gained from the visualization.

- Finally, to determine the success of the visualization.

North (2006) argues that if the purpose of visualization is to give insight, then the purpose of visualization evaluation is to determine the extent to which the visualization achieves this. The focus is on the team-evaluation of the VM to determine its suitability in a given context, rather than 
on evaluation of the VM per se. This contrasts with the conventional approach that evaluates a visualization method in and of itself.

Where two or more methods are used to visualize the specific data set, Wiss, Carr and Jonsson (1998) recommend evaluation by comparison to determine which VM is most suitable and which better supports the required user tasks. The visualization should be evaluated to ensure that the required insight has been generated, and that the insight has been acquired by the user. This can be identified and measured using the insight-based methodology of Saraiya, North and Duca (2005). The authors of the approach warn that it is labour-intensive and time-consuming; therefore it is not included in the current version of RoutePlanner. A subset, namely the scheme to capture and to code the quantifiable characteristics of each insight is, however, included. As a further approach, the overall success of the visualization can be evaluated using the factors proposed by Kao and Ma (2000). This is a less formal technique than identifying insight.

Figure 6 presents a diagrammatic representation of the evaluation stage, showing the respective evaluation approaches of Wiss et al (1998), Saraiya et al (2005), and Kao and Ma (2000).

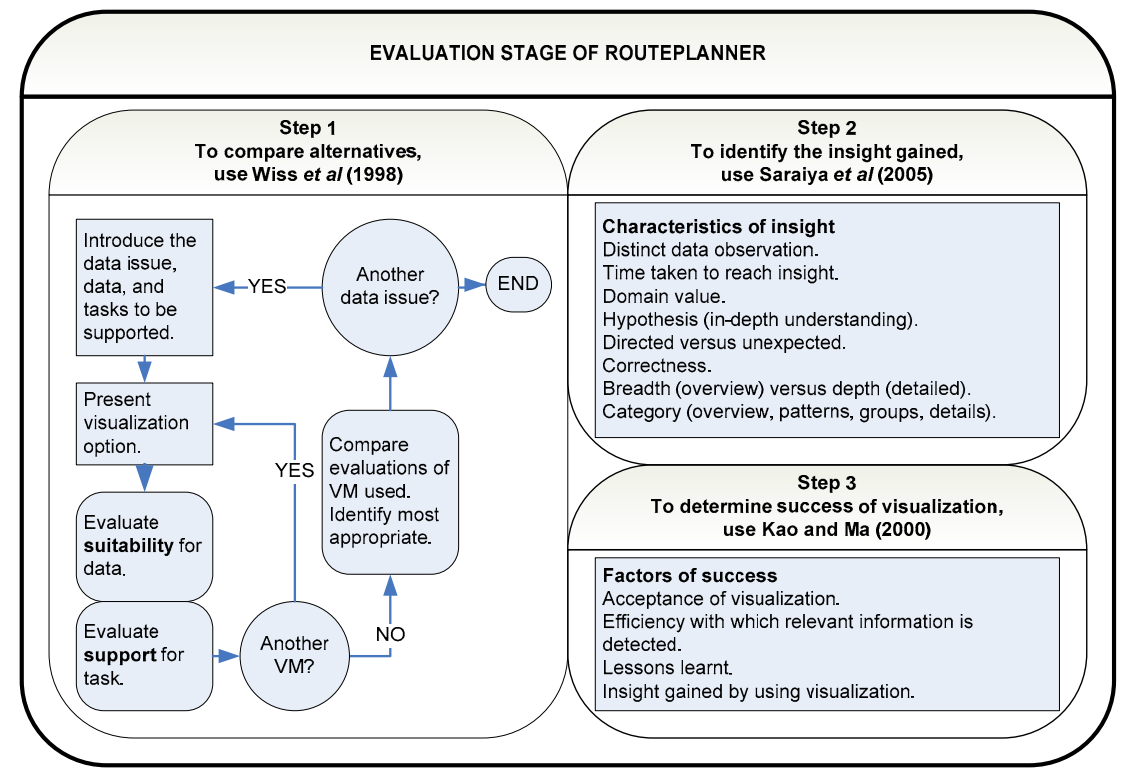

Figure 6 Evaluation stage of RoutePlanner

Evaluation is the final stage of RoutePlanner's visualization process. If users have not gained the required insight as per the insight-based methodology, or if the visualizations are not deemed successful in line with the factors of Kao and Ma (2000), then a return to the selection stage, followed by another evaluation stage, may be necessary.

\section{Evaluation of the RoutePlanner Model}

This empirical work mentioned in the research design section, was conducted in small-group collaborative sessions, during which the model was introduced and demonstrated, and was used by the groups of brokers participating in the study.

\section{Validating the Need for Visualization}

To validate the need for, and the value of, visualization, a questionnaire was used to collect data from the participants, heterogeneous with respect to age, gender, and experience, to establish their awareness of visualization before and after exposure to RoutePlanner. Six statements were rated on the 5-point Likert scale, where 1 represents strongly disagree and 5 represents strongly agree. 
Figures 7 and 8 respectively show the percentages obtained before and after exposure to RoutePlanner for the following six statements as they appeared in the questionnaire:

- I know the details of the process that is required to transform raw into visualized data.

- I know how to identify the data to be visualized.

- I know how to select appropriate visualization methods.

- I know how to evaluate visualizations of required data.

- I am aware of the benefits of the visualization of data.

- I am aware of the insights that may be gained from the visualization of the data.

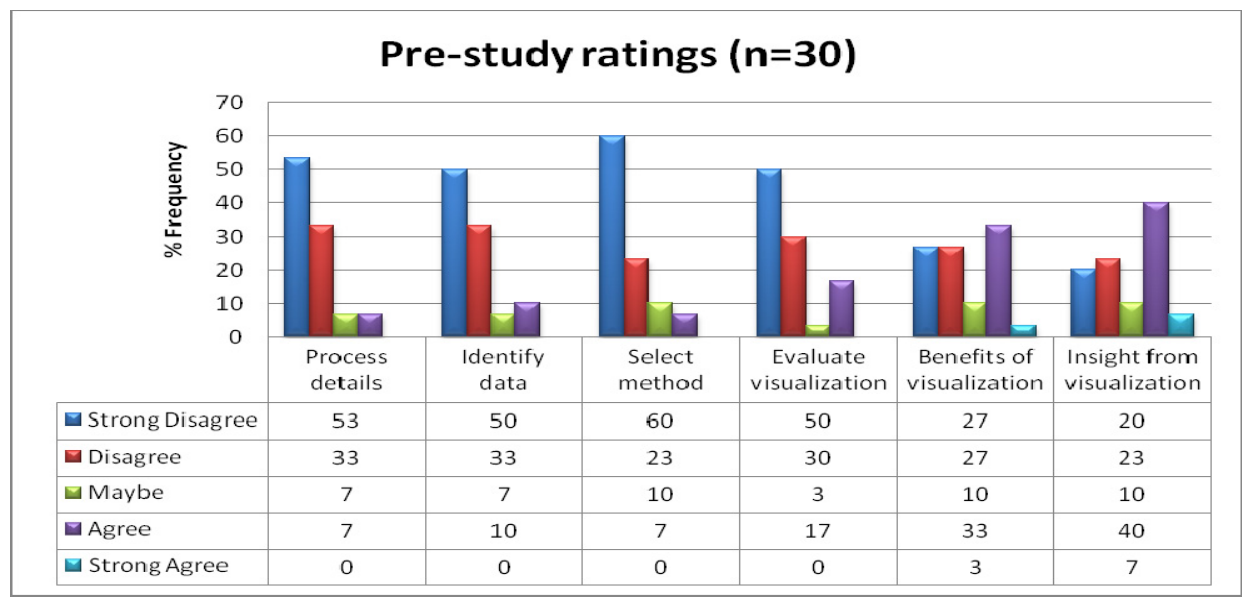

Figure 7 Pre-study ratings $(\mathbf{n}=\mathbf{3 0})$

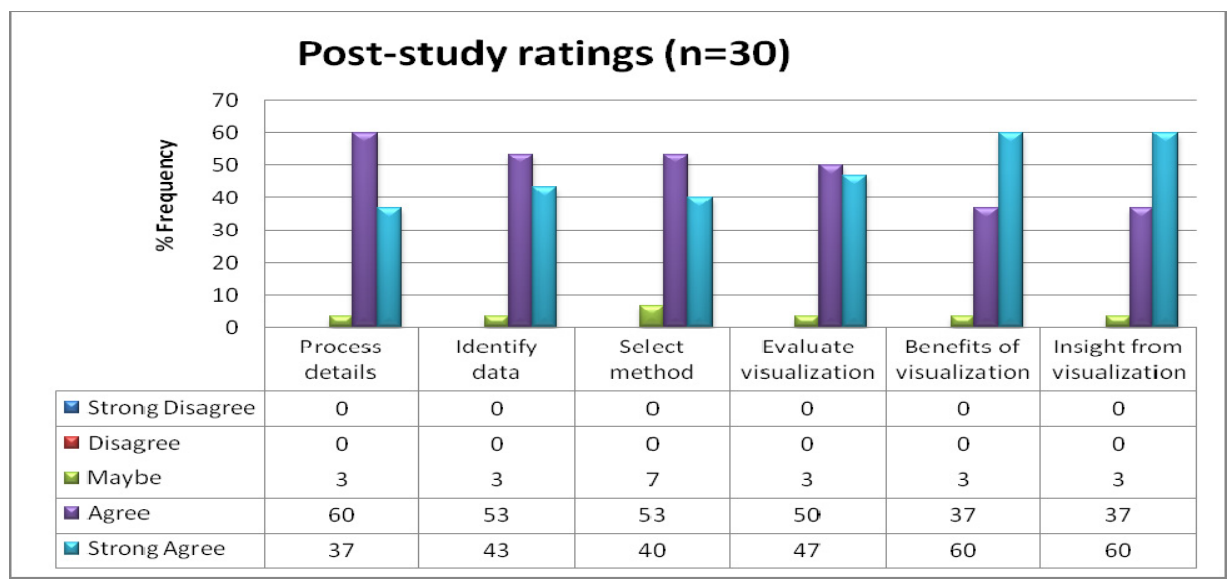

Figure 8 Post-study ratings $(n=30)$

These results show that, although the participants were largely unfamiliar with details of visualization processes and visualization methods prior to the orientation sessions (only between $7 \%$ and $17 \%$ agreed with the statements), they were relatively aware of the benefits of visualization and the insights to be gained if they used IV in interpreting their client data (36\% and $47 \%$ agreed with the statements).

Thus, it can be concluded that, although the participants were largely unaware of the details of the visualization process, as addressed by the first four statements in the questionnaires (average ratings 1.7, 1.8, 1.6 and 1.9), they were relatively aware of the benefits of the visualization and the insights to be gained from using it in interpreting their client data (average ratings of the last two statements: 2.6 and 2.9). 
After exposure to RoutePlanner, the awareness levels increased. The participants were familiar with details of visualization processes and the selection of visualization methods after the orientation sessions (between 93\% and 97\% agreed with the statements), and they were more aware of the benefits of visualization and the insights to be gained if they used IV in interpreting their client data (97\% and $97 \%$ agreed with the statements).

Most participants were acutely aware of the details of the visualization process (average ratings 4.3, 4.4, 4.3 and 4.4 for the first four statements) and had a greater awareness of its benefits, as well as the insights to be gained from the use of visualization in investigating their client data (average ratings 4.6 and 4.6 for the last two statements). The percentages in Figure 8 show a completely different picture from Figure 7, representing the increase in awareness of the visualization process and the benefits of its use.

The Cochran-Armitage test was used to test for trend analysis of the data. Item analysis was used in the calculation of a general awareness score for the pre- and the post-exposure data for each participant. The differences between the two sets of scores were proven statistically significant.

\section{Evaluation of the Model Itself}

During the session, an evaluation study was conducted to assess utility and relevance of each aspect of RoutePlanner. In open-ended responses, participants noted various positive and negative features; they used the opportunity to suggest modifications and to indicate the relevance of that aspect to their professional tasks in the workplace. The ratings of each aspect were as follows:

Guidelines: Each set of practical guidelines for visualization was evaluated, Brath's (1999) guidelines, the environmental factors of Mann (1999) and Mann \& Reiterer (2000), the universal visualization issues of Spence (2001), and the trends in visualization. In each case, responses were positive with the majority of participants agreeing or strongly agreeing that they could use the guidelines. The percentage frequencies of 'Agree' and 'Strongly agree' varied from $67 \%$ to 97\%. Eighty three percent ( $83 \%$ ) identified positive features and $43 \%$ ) noted negative features related to the utility of the guidelines or to a lack of familiarity. Seven percent (7\%) suggested that features be removed and $17 \%$ suggested other alternatives, thus a total of $24 \%$ proposed modifications. Eighty three percent (83\%) indicated that the guidelines were indeed relevant to their workplace activities and facilitated the process of visualizing their data.

Identification stage: Ninety seven percent (97\%) of participants agreed or strongly agreed with the statements relating to the identification of data for visualization and the associated tasks for the user. Seventy seven percent (77\%) identified positive features focused on utility and functionality, while only $10 \%$ mentioned negative features and $7 \%$ suggested certain modifications. The majority (93\%) indicated that this stage was relevant to their work. It was clear that the identification stage of RoutePlanner can be used successfully by the target group.

Selection stage: Ninety seven percent (97\%) of participants agreed or strongly agreed with the statements relating to the selection of appropriate visualization methods for the tasks. Seventy seven percent (77\%) identified positive features, while only $10 \%$ mentioned negative features and $7 \%$ suggested possible modifications. The majority (93\%) found this stage highly relevant to their work. It was evident that they were learning to use the processes included in RoutePlanner.

Evaluation stage: More than ninety percent (93\%) agreed or strongly agreed that the processes for comparing visualizations and identifying the insight gained were effective, while $97 \%$ either agreed or strongly agreed that the factors to determine success of visualization or lack of success, can be applied. Seventy six percent (76\%) focused on the positive features, $3 \%$ noted negative features, $3 \%$ suggested modifications and $87 \%$ found the evaluation stage relevant to their work. It can therefore be concluded that the participants were orienting themselves accurately on the 
RoutePlanner journey and that they were able to use its evaluation stage. Furthermore, given the implementation of the evaluation stage, as undertaken during the small-group sessions, all the visualizations implemented during the intervention were deemed successful.

\section{Conclusion}

The study set out to answer the following questions, which we now re-visit:

Does the system currently used by the target group of brokers support users in translating raw data into insight? From the identification-of-need study, it was concluded that more than $80 \%$ of the participants either agreed or strongly agreed with the statements relating to a need for visualization, a preference for familiar visualizations, a keenness to learn about visualization, as well as an awareness of the economic benefits of using visualization. This need is not met by the FNA system currently used by the brokers.

What are appropriate stages in the visualization process of translating data into insight and what should each stage entail? The stages of the visualization process and their details were investigated by in-depth studies of relevant literature, which revealed that there is no single definition or absolute set of stages in the visualization process as a path from raw data to required insight. However, a consolidated model, RoutePlanner, was formulated for the visualization process. This model takes account of many definitions and structures from the literature and decomposes the process into three generic stages, namely identification, selection and evaluation. The completion of each stage leads to the commencement of the next.

What guidelines can help users apply the visualization process? Guidelines were identified for the application of this visualization process. The collection of guidelines is relevant to all three stages of the process. They are the most appropriate starting point prior to the use of RoutePlanner, since completion of the guideline templates should enhance the overall focus on the visualization of the relevant data. The guidelines comprise a body of knowledge that can be used by domain experts and data experts to support meaningful generation and interpretation of visualizations, and can also be used by visualization experts for reference and leadership.

Can this visualization process be used by brokers to gain adequate insight? This question consists of two parts, namely, whether or not the visualization process can be used and, if it can be used; whether brokers can use it enhance insight to such an extent that it improves their performance in their professional activities? The evaluation of RoutePlanner revealed that the model has utility and that all of its stages, as well as RoutePlanner as a whole, are relevant to the work of the financial brokers, and that all visualizations were successful in terms of the enhanced insight acquired. With the selected visualizations and the associated new insight, brokers are better informed, and they in turn can better inform and advise their clients during financial planning sessions.

With regard to the collaborative approach between domain expert, data expert and visualization expert to foster the learning and application of RoutePlanner, over $70 \%$ of the participants who responded to the relevant question, preferred the supportive team approach to orientation, rather than a solo approach. It is anticipated that, in due course, as technological expertise and exposure to visualization increase, as further training and orientation are conducted and, ultimately, when a visualization system is developed comprising visualization methods customized to the domain of insurance brokerage, then brokers will be able to use visualization processes independently.

This study relates to an initial version of RoutePlanner. It is likely that as the body of visualization knowledge evolves so too, will RoutePlanner. The study makes a contribution to the focus area of the process of information visualization. RoutePlanner, as a model of the visualization process, is an innovative supportive framework that integrates multiple aspects of the visualiza- 
tion process, such as existing guidelines, approaches in the body of visualization knowledge, and public-domain visualization resources.

\section{References}

Brath, R. K. (1999). Effective information visualization: Guidelines and metrics for 3D interactive representations of business data. Unpublished MA. (Computer Science). Toronto: University of Toronto.

Card, S. K., Shneiderman, B., \& Mackinlay, J. D. (1999). Readings in information visualization: Using vision to think. San Francisco, Calif.: Morgan Kaufmann Publishers.

de Oliveira, M. C. F., \& Levkowitz, H. (2003). From visual data exploration to visual data mining: A survey. IEEE Transactions on Visualization and Computer Graphics, 9(3): 378-394.

Duke, D. J., Brodlie, K. W., Duce, D. A., \& Herman, I. (2005). Do you see what I see? IEEE Computer Graphics and Applications, 25(3): 6-9.

Espinosa, O. J., Hendrickson, C., \& Garrett, J. H. (1999). Domain analysis: A technique to design a usercentered visualization framework. IEEE Symposium on Information Visualization (Info Vis '99): 44-52, 144. Washington, D.C, USA: IEEE Computer Society Press.

InfoVis ToolKit (2008) The InfoVis ToolKit. Retrieved 2008/12/19 from http://ivtk.sourceforge.net/

Kao, D. L., \& Ma, K. L. (2000). The life cycle of a visualization case study. IEEE Computer Graphics and Applications, 20(5), 29-31.

Keim, D. A. (2002). Information visualization and visual data mining. IEEE Transactions on Visualization and Computer Graphics, 8(1), 1-8.

Kimball, R. (1996). The data warehouse toolkit: Practical techniques for building dimensional data warehouses. New York: John Wiley \& Sons.

Kimball, R., Reeves, L., Ross, M., \& Thornthwaite, W. (1998). The data warehouse lifecycle toolkit: Expert methods for designing, developing, and deploying data warehouses. New York: Wiley.

Lengler, R., \& Eppler, M. J. (2007). Towards a periodic table of visualization methods for management. In Graphics and Visualization in Engineering (GVE2007): 1. Calgary, AB: Acta Press.

Mann, T. M. (1999). Visualization of WWW search results. Proceedings of the 10th International Workshop on Database and Expert Systems Applications (DEXA '99): 264. Washington, DC, USA: IEEE Computer Society.

Mann, T. M., \& Reiterer, H. (2000). Evaluation of different visualization of www search results. Proceedings of the 11th International Workshop on Database and Expert Systems Applications: 586. Washington, D.C., USA: IEEE Computer Society.

Mouton, J. (2003). How to succeed in your master's and doctoral studies: A South African guide and resource book. Pretoria: Van Schaik Publishers.

North, C. (2006). Toward measuring visualization insight. IEEE Computer Graphics and Applications, 26(3), 6-9.

OLIVE (1997) On-line library of information visualization environments. Retrieved 2008/11/18 from http://otal.umd.edu/Olive/

Saraiya, P., North, C., \& Duca, K. (2005). An insight-based methodology for evaluating bioinformatics visualizations. IEEE Transactions on Visualization and Computer Graphics, 11(4), 443-456.

Shneiderman, B. (1996). The eyes have it: A task by data type taxonomy for information visualizations. Proceedings of the 1996 IEEE Symposium on Visual Languages (VL '96): 336. Washington, DC, USA: IEEE Computer Society.

Spence, R. (2001). Information visualization. Essex, England: Addison-Wesley.

van Wijk, J. J. (2006). Bridging the gaps. IEEE Computer Graphics and Applications, 26(6), 6-9. 
Wiss, U., Carr, D. A., \& Jonsson, H. (1998). Evaluating three-dimensional information visualization designs: A case study of three designs. Proceedings of the International Conference on Information Visualisation (IV'98): 137. Washington, DC, USA: IEEE Computer Society.

VisTree (2008). The Visualization Tree. Retrieved 2008/12/19 from http://gbdi.icmc.usp.br/ junio/VisTree/Conteudo/Download.htm

XmdvTool (2008) Interactive visual exploration of multivariate data. Retrieved from 2008/12/19 http://davis.wpi.edu/ $\sim \mathrm{xmdv} /$

\section{Biography}

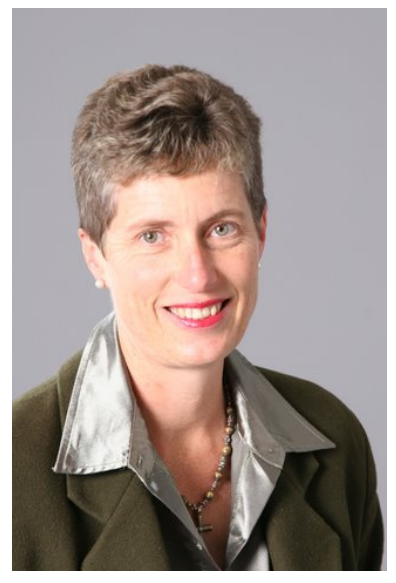

Patricia Gouws is a lecturer in the School of Computing at the University of South Africa (UNISA), a distance-teaching institution. Patricia has an MSc in information systems, focusing on visualization of data, and BSc (Honours) degrees in both Information Systems and Operational Research. She teaches visual programming to all undergraduate levels, and coaches a First Lego League team as part of her community engagement responsibilities.

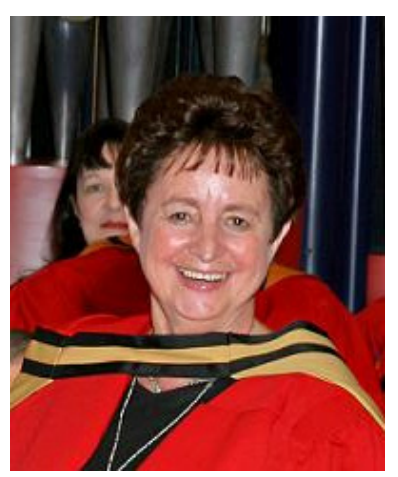

Ruth de Villiers is a professor in the School of Computing at the University of South Africa (UNISA), a distance-teaching institution, which emphasizes the role of research into open distance learning and educational technology. Ruth has a PhD and also holds masters degrees in the domains of Information Systems and Computer-integrated Education, respectively. For more than twenty five years, she has taught Computer Science and Informatics. Her major current research interests and teaching areas are Human-Computer Interaction and eLearning. She has combined these areas by undertaking research and development in the usage, usability and usability evaluation of a broad variety of e-learning applications and other environments. She has also published in the focus area of meta-research, involving work on various research designs and methodologies. Ruth supervises master's and doctoral students in the fields mentioned above. 